\title{
Developmental stuttering and Parkinson's disease: the effects of levodopa treatment
}

\author{
Jeffrey M Anderson, John D Hughes, Leslie J Gonzalez Rothi, Gregory P Crucian, K M \\ Heilman
}

\begin{abstract}
The effects of dopamine on developmental stuttering was studied in a 44 year old man with developmental stuttering and Parkinson's disease during three levodopa "on" periods and three "off" periods. When compared with the "off" periods, during the "on" periods he demonstrated an increase of speech dysfluencies. These findings lend support to the dopamine hypothesis of developmental stuttering.

(F Neurol Neurosurg Psychiatry 1999;66:776-778)
\end{abstract}

Keywords: stuttering; Parkinson's disease; dopamine agonists

The cause of developmental stuttering is unknown; however, the neuroleptic drug haloperidol decreases speech dysfluencies. ${ }^{1-3}$ The efficacy of haloperidol may be due to its psychotrophic effects or an alteration of the underlying pathophysiological mechanisms. Haloperidol blocks dopaminergic receptors, D2 more than D1, and developmental stuttering might be related to a hyperactive dopaminergic state. $^{24}$

Patients with Parkinson's disease often show a hypokinetic dysarthria, ${ }^{5}$ hypophonia, and monotonous pitch, but typically they do not stutter. However, palilalia, another disorder of speech fluency, may be associated with Parkinson's disease. ${ }^{6}$ Developmental stuttering, unlike palilalia, is associated with speech repetitions of the initial phonemes and also cooccurs with speech blocks. ${ }^{7}$ The patient we report on had developmental stuttering, but did not show palilalia.

Levodopa treatment relieves many of the symptoms of Parkinson's disease. Dopaminergic treatment seems to increase motor activation and it might be expected that levodopa treatment would increase speech fluency or have no effect. We report on a patient with developmental stuttering and Parkinson's disease that provided us an opportunity to study the effects of levodopa on speech fluency during "on" and "off" periods.

\section{Case report}

A 44 year old right handed man was referred for the pharmacological management of his Parkinson's disease that included rigidity, bradykinesia, akinesia, and a resting tremor. His Parkinson's disease was somewhat atypical because it started at the age of 31 and at the time of testing he was unable to walk without support. He also reported a lifelong history of developmental stuttering that had begun during early childhood. The patient reported no family history of movement disorders. At the time of testing he was on a regiment of carbidopa-levodopa (25/100) three times a day (5 $00 \mathrm{am}, 1000 \mathrm{am}$, and $300 \mathrm{pm}$ ). Behaviourally during carbidopa-levodopa treatment the patient oscillated between "on" periods characterised by moderate/severe stuttering and dyskinesias, but with mild or little bradykinesia, tremors, or rigidity, and "off" periods with mild stuttering, no dyskinesias, but with severe resting tremors (more severe distally than proximally), akinesia, bradykinesia, and rigidity. Because he was unable to walk and was confined to a wheelchair when either "on" or "off" levodopa, his disability was graded as a stage $\mathrm{V}$ using the Hoehn and Yahr criteria. ${ }^{8}$ During testing, all "on" sessions occurred about 1 hour after a dose of carbidopalevodopa and all "off" sessions occurred no sooner than 4 hours after the last dose of carbidopa-levodopa.

The patient reported an automobile accident 26 years before our testing in which he may have had a brief loss of consciousness. However, his stuttering predated his accident and his speech dysfluencies did not increase or decrease after the accident. Recent MRI demonstrated only mild cortical atrophy.

\section{Methods}

ANALYSIS OF SPEECH DYSFLUENCIES

The patient's speech was tested during six separate sessions; three "on" periods, and three "off" periods. The "on" and "off" sessions varied from morning to afternoon. The same examiner, a speech-language pathologist, tested the patient during these six occasions.

The examiner asked the subject to tell common fairy tales randomly selected from the following list; Cinderella, Little Red Riding Hood, Jack and the Beanstalk, or Pinocchio. The sessions were audiotaped and a written transcript was created from these tapes. Two raters were provided with the written transcript, listened to the audiotapes of the "on" 
and "off" sessions (in an randomised order) and marked on the transcript each dysfluent episode. These raters were unaware or blinded to the patient's clinical state and the research question. The raters computed the total number of dysfluencies produced per session as well as the type of speech dysfluencies produced by the subject including speech blocks, initial phoneme repetitions, and the presence of extraneous interjections or filler words. These dysfluencies were totalled for each rater and the scores for each session were averaged. A speech-language pathologist compiled the number of syllables produced during each session and the rate of dysfluencies produced for each 100 syllables was calculated. A speech block was defined as a significant, non-natural pause or interruption. An initial phoneme repetition was defined as the repeated production of the initial speech sound. A filler word was defined as an extraneous interjection such as "ah".

\section{Results}

The interrater agreement between the two judges for the overall average rate of speech dysfluencies produced per minute during "on" and "off" sessions was greater than $90 \%$ $(r=0.91)$. A non-parametric Friedman test was used to compare the dysfluency rate per 100 syllables. The dysfluency rate during "on" session 1 was significantly different when compared to the "off" session 1 rate $(p<0.05)$. The "dysfluency rate per 100 syllables during "on" session 2 when compared to "off" session 2 was not significantly different $(p=0.31)$, and the dysfluency rate produced during "on" session 3 was also not significantly different $(p=1.00)$ from those produced during "off" session 3 . The average rate of the production of words per minute for the three "on" sessions was 73.2 (SEM 5.3), whereas during the three "off" sessions the average rate was 73.1 (SEM 8.8). A paired $t$ test of the rate of words produced each minute during the three "on" sessions, was not significantly different $(p=0.99)$ from the rate during the three "off" sessions.

\section{Discussion}

These results suggest that our patient's stuttering was more severe with carbidopa-levodopa treatment. Although his average rate of word production remained the same during both "on" and "off" sessions, during "on" session 1 the patient showed severe stuttering as evidenced by significant increases in the number of sound repetitions, speech blocks, and use of filler words when compared with "off" session 1. The subject's performance across the six trials displayed an adaptation effect (figure), in that the repeated production of the same passages was associated with a reduction in the overall number of speech dysfluencies across trials. This adaptation effect is characteristic of developmental stuttering. ${ }^{7}$

Koller reported six patients with extrapyramidal disease and dysfluent speech. ${ }^{9}$ In one case Koller noted that the patient had an "onoff” phenomena. ${ }^{9}$ Although not formally tested

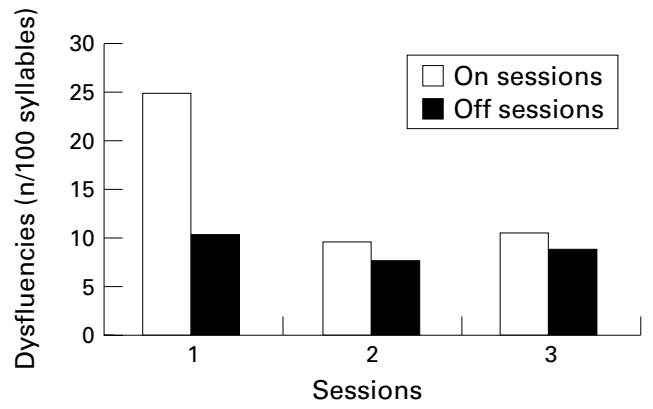

Dysfluencies produced during on and off levodopa sessions.

this patient's stuttering seemed to be worse during his "on" periods than it was in his "off" periods.

The neuromuscular activities that support speech production are complex and the mechanisms inducing stuttering are not entirely known. Nudelman et al in an engineering based, ${ }^{10}$ motor control theory of speech production, propose the existence of two main nested feedback loops, an outer "linguistic loop" that decides and monitors what sounds are to be made, and an inner "phonatory loop" that is involved with motor programming of the vocal apparatus. Nudelman et $a l^{10}$ argue that stuttering can be modelled as a momentary instability in the system whenever the timing between these two loops is momentarily disrupted. Although this model is not anatomically based, the outer linguistic loop is likely supported by cortical areas in the dominant hemisphere's perisylvian areas, whereas the inner phonatory loop is likely supported by the dominant hemisphere's frontal cortex, supplementary motor area, and striatum via corticostriatothalamocortical circuits. ${ }^{10} 11$

Nudelman et $a l^{12}$ have suggested that the neural processing in the outer linguistic loop (likely mediated by language cortex) is slowed in stutterers. In the absence of adequate dopamine there is putamenal dysfunction. The putamenal dysfunction associated with Parkinson's disease may slow down the cortical basal ganglia circuit supporting speech production. The most commonly occurring motor speech disorder in Parkinson's disease is hypokinetic dysarthria marked by a decrease in the amplitude of orolingual muscular movements and decreased respiratory support. ${ }^{13}{ }^{14}$ As a result "speech hastening" may occur that is analogous to a festinating petit pas gait of Parkinson's disease. Speech hastening is associated with movements of the articulators producing poorly articulated, but accelerated speech.

Nudelman et $a l^{12}$ posit that in nonParkinsonian developmental stutterers, the outer linguistic loop is slower than the inner phonatory loop. Perhaps the palilalia associated with Parkinson's disease is induced by a slowing of the inner phonatory loop in reference to the outer linguistic loop.

By contrast with patients with palilalia, perhaps during this patient's "on" sessions, while the outer loop remains slowed, the inner phonatory loop becomes accelerated, due to the putamenal receptors receiving more dopamine, resulting in speech dysfluencies. 
However, during "off” sessions his baseline speech dysfluencies, although still present, are diminished because with reduced dopamine the subcortical inner phonatory loop is slowed and therefore less out of synchrony with the slowed outer linguistic loop.

Dopamine blockers such as haloperidol may also slow the inner phonatory loop and help stutterers to re-establish synchrony. Unfortunately haloperidol was not well tolerated by stutterers. ${ }^{23}$ However, more recent dopamine antagonists such as risperidone or olanzapine, with fewer side effects than haloperidol, may provide a tolerable treatment option for developmental stutterers.

This work was supported by the Veterans Administration Research Service. We thank Dr Kenneth Logan for information concerning the assessment of dysfluencies.

1 Brady JP. The pharmacology of stuttering: a critical review. Am F Psychatry 1991;148:1309-16.

2 Rosenberger PB, Wheelden JA, Kalokin M. The effect of haloperidol on stuttering. Am f Psychiatry 1976;133:331-4
3 Burns D, Brady JP, Kuruvilla K. The acute effect of haloperidol and apomorphine on the severity of stuttering. Biology Psychiatry 1978;13:255-64.

4 Waddington JL, O-Boyld KM. Drugs acting on brain dopamine receptors. Pharmacol Ther 1989;43:1-52.

5 Illes J, Metter EJ, Hanson WR, et al. Language production in Parkinson's disease: acoustic and linguistic considerations. Brain Lang 1988;33:146-60.

6 Boller F, Albert M, Denes F. Palilalia. Br f Disord Commun 1975;10:92-7.

7 Bloodstein O. A handbook on stuttering. San Diego, CA: Singular Publishing, 1995.

8 Hoehn MM, Yahr MD. Parkinsonism: onset, progression and mortality. Neurology 1967;17:427-42.

9 Koller WC. Dysfluency (stuttering) in extrapyramidal disease. Arch Neurol 1983;40:175-7.

10 Nudelman HB, Herbrich BD, Hoyt BD, et al. A neuroscience model of stuttering. Fournal of Fluency Disorneuroscience model of
ders $1989 ; 14: 399-427$.

11 Alexander GE, DeLong MR, Strick PL. Parallel organization of functionally segregated circuits linking basal ganglia and cortex. Annu Rev Neurosci 1986;9:357-81.

12 Nudelman HB, Herbrich BD, Hess KR, et al. A model of phonatory response time of stutters and fluent speakers to frequency-modulated tones. I Acoust Soc Am 1992;92: 1882-8.

13 Hammen VL, Yorkston KM. Speech and pause characteristics following speech rate reduction in hypokinetic dysarthria. F Commun Disord 1996;29:429-44.

14 Solomon NP Hixon TJ. Speech breathing in Parkinson's disease. F Speech Hear Res 1993;36:294-310. 